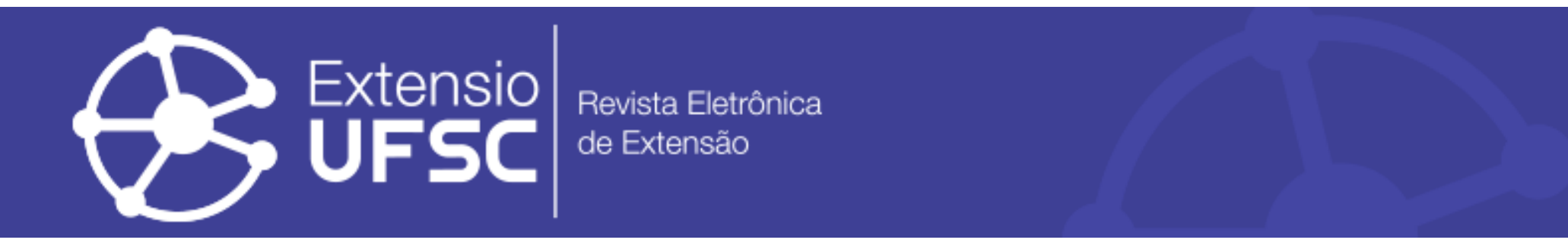

\title{
ATENDIMENTOS NUTRICIONAIS AMBULATORIAIS E ESTADO NUTRICIONAL DE CRIANÇAS MENORES DE SEIS ANOS
}

\author{
Pâmela Schactae Lacerda \\ Universidade Estadual do Centro-Oeste \\ pamelaschactae_22@hotmail.com \\ Nilciane Taques \\ Universidade Estadual do Centro-Oeste \\ nilcianetaques@hotmail.com \\ Jéssica Patricia Cain \\ Universidade Estadual do Centro-Oeste \\ jessi.graff@hotmail.com
}

\author{
Luana Molinari \\ Universidade Estadual do Centro-Oeste \\ luanamolinari15@gmail.com \\ Caryna Eurich Mazur \\ Universidade Estadual do Centro-Oeste \\ carynanutricionista@gmail.com \\ Paula Chuproski Saldan \\ Universidade Estadual do Centro-Oeste \\ pchuproski@unicentro.br
}

\section{Resumo}

Objetivo: Descrever atendimentos nutricionais prestados às crianças menores de seis anos pelo projeto de extensão no Centro de Recuperação Nutricional de Guarapuava-PR. Método: Os atendimentos foram efetuados semanalmente por discentes e docentes do curso de Nutrição no período de 2015-2017. Realizava-se avaliação antropométrica das crianças (peso e estatura), anamnese para conhecer mães e crianças e avaliação do consumo alimentar. Após o diagnóstico nutricional eram repassadas orientações nutricionais. Eram agendadas consultas mensais para crianças com comprometimento do estado nutricional. Atividades de Educação Alimentar e Nutricional (EAN) foram desenvolvidas para auxiliar as mães na alimentação das crianças. Resultados: Realizou-se 133 atendimentos e a maioria na faixa etária de 623 meses (n=81). A maioria das crianças apresentou estado nutricional adequado (62\%). As atividades de EAN foram oficina culinária e roda de conversa. Conclusões: Apesar da maioria das crianças estar eutrófica, observou-se que 38\% tinham comprometimento do estado nutricional, sendo fundamental seu acompanhamento.

Palavra-chave: Assistência Ambulatorial. Antropometria. Estado Nutricional. Educação Alimentar e Nutricional.

\section{OUTPATIENT NUTRITIONAL APPOINTMENTS AND NUTRITIONAL STATUS OF CHILDREN UNDER THE AGE OF SIX}

Abstract

Objective: To describe the outpatient nutritional appointments provided to children under the age of six through an extension project at the Nutritional Recovery Center in Guarapuava-PR. Method: The appointments were carried out weekly by students and professors of the Nutrition course in the period 2015-2017. An anthropometric evaluation of children (weight and height), anamnesis to acquaint mothers and children and evaluation of food consumption were made. After the nutritional diagnosis, nutritional guidelines were given to mothers. Monthly consultations were scheduled for children with impaired nutritional status. Food and Nutrition Education (FNE) activities were developed to assist mothers in feeding children. Results: A total of 133 attendances were carried out and the most appointments were in the age range of $6-23$ months $(n=81)$. The majority of children presented adequate nutritional status $(62 \%)$. The FNE activities were culinary workshop and conversation exchange. Conclusions: Although the majority of children were eutrophic, $38 \%$ had some impairment of the nutritional state, being essential their monitoring.

Keywords: Ambulatory Care. Anthropometry. Nutritional Status. Food and Nutrition Education.

\section{ATENCIÓN AMBULATORIA NUTRICIONAL Y ESTADO NUTRICIONAL DE NIÑOS MENORES DE SEIS AÑOS}

\section{Resumen}

Objetivo: Describir atendimientos nutricionales prestados a los niños menores de seis años por el proyecto de extensión en el Centro de Recuperación Nutricional de Guarapuava-PR. Método: Los atendimientos fueron realizados semanalmente por discentes y docentes del curso de Nutrición en el periodo 2015-2017. Se realizaba evaluaciones antropométricas en los niños (peso y estatura), anamnesis para conocer a las madres y niños y evaluación del consumo alimentar. Después del diagnóstico nutricional fueron repasadas las orientaciones nutricionales. Además fueron programadas consultas mensuales para los niños con comprometimiento en el estado nutricional. Actividades de Educación Alimentaria y Nutricional (EAN) fueron desarrolladas para auxiliar a las madres en la alimentación de los niños. Resultados: Fueron realizados 133 atendimientos, los mayores fueron en el grupo de 6-23 meses ( $\mathrm{n}=81$ ). La mayoría de los niños presentaron el estado nutricional adecuado (62\%). Las actividades de EAN fueron taller culinario y rueda de conversación. Conclusiones: A pesar de que la mayoría de los niños estaban eutróficos, fue observado que 38\% tenían algún comprometimiento del estado nutricional, siendo fundamental su acompañamiento.

Palabras clave: Atención Ambulatoria. Antropometría. Estado Nutricional. Educación Alimentaria y Nutricional. 
Atendimentos nutricionais ambulatoriais e estado nutricional de crianças menores de seis anos

\section{INTRODUÇÃO}

A primeira infância compreende o período dos 0 aos 6 anos de vida da criança. $O$ desenvolvimento integral saudável nessa fase da vida com nutrição e cuidados de saúde adequados, ambiente familiar afetivo, seguro e estimulante, relações estáveis e educação de qualidade fornece a base para que a criança atinja seu potencial pleno na vida adulta (COMITÊ CIENTÍFICO DO NÚCLEO CIÊNCIA PELA INFÂNCIA, 2014).

O estado nutricional (EN) de uma criança é um importante indicador de sua saúde global e os processos de crescimento e desenvolvimento infantil associam-se fortemente as condições de vida. Problemas relacionados à nutrição de crianças podem trazer repercussões em curto e longo prazo já que este período se caracteriza pelo acelerado crescimento corporal que impõe grandes necessidades nutricionais reforçando a importância de acompanhar o EN nesta fase da vida, para que sejam articuladas atividades de prevenção e intervenção adequadas (DUNCAN; SCHMIDT; GIUGLIANI, 2004; NASSER, 2006).

Os déficits de crescimento na infância, independente dos fatores etiológicos, estão associados a um aumento significativo de riscos de mortalidade, aparecimento de doenças infecciosas, prejuízos no desenvolvimento psicomotor, baixo aproveitamento escolar e menor capacidade produtiva na fase adulta. Por outro lado, hábitos alimentares inadequados desde o início da vida podem levar a carências nutricionais, desnutrição e sobrepeso, condições que preocupam por serem preditoras de obesidade, além de estarem associadas com o surgimento de outras doenças crônicas não-transmissíveis (MELO; LUFT; MEYER, 2004; BLACK et al., 2008; BRASIL, 2012).

As orientações nutricionais propostas para crianças assumem um papel imprescindível como garantia do desenvolvimento satisfatório, introduzindo os alimentos adequadamente em idade correta e propiciando meios para que possam atingir o seu potencial biológico. São também grandes estratégias na construção dos hábitos alimentares e promoção de estilos de vida saudáveis (SIGULEM; TADDEI, 2004; VIEIRA et al., 2009). Dentre as ações está o acompanhamento do crescimento e desenvolvimento das crianças por meio de atendimentos nutricionais. No acompanhamento do crescimento se faz a avaliação periódica do ganho de peso, estatura, sendo possível avaliar o progresso da criança, identificando aquelas em maior risco de morbimortalidade, prevenindo precocemente inadequações do EN e promovendo o crescimento infantil de forma saudável e adequada (CARVALHO et al., 2008).

O objetivo deste trabalho é descrever atendimentos ambulatoriais em Nutrição prestados a crianças menores de seis anos, por meio de um projeto de extensão em um Centro de 
Atendimentos nutricionais ambulatoriais e estado nutricional de crianças menores de seis anos

Recuperação Nutricional de Guarapuava-PR. Além disso, relatar sobre o estado nutricional das crianças no momento inicial dos atendimentos.

\section{MATERIAIS E MÉTODOS}

Trata-se de um projeto de extensão por tempo determinado que acontece por meio de atendimentos nutricionais ambulatoriais em um Centro de Recuperação Nutricional da cidade de Guarapuava-PR. O projeto está vinculado ao Departamento de Nutrição da Universidade Estadual do Centro-Oeste (UNICENTRO) e vem sendo desenvolvido desde 2009 por docentes e discentes do curso de Nutrição. A cada dois anos acontece a renovação do projeto e da equipe de discentes, em função do tempo máximo de duração dos projetos institucionais de extensão (24 meses). Será relatado como aconteceram esses atendimentos referentes aos últimos dois anos do projeto (maio de 2015 a abril de 2017) e a situação do estado nutricional, segundo o índice antropométrico Peso/Idade, das crianças atendidas até setembro de 2016.

Os atendimentos nutricionais aconteceram semanalmente (quintas-feiras) a partir das 13:30 até 17:00 horas, sendo realizado no máximo oito atendimentos diários. Primeiramente, era realizada a avaliação antropométrica das crianças, com aferição de peso e estatura e classificação do EN segundo as curvas de crescimento da Organização Mundial de Saúde (OMS) (2006/2007) com base nos índices Peso para Idade (P/I), Estatura para Idade (E/I) e Índice de Massa Corporal para Idade (IMC/I) expressos em percentis (BRASIL, 2011). Para crianças prematuras, com Síndrome de Down e Paralisia Cerebral foram utilizadas curvas específicas (MUSTACCHI, 2002; BROOKS et al., 2011; FENTON; KIM, 2013).

Após a avaliação antropométrica das crianças, iniciavam-se os atendimentos individuais que eram realizados por ordem de chegada. Nesse atendimento era aplicada uma anamnese nutricional para caracterização das mães e crianças com base na descrição do sexo, faixa etária, peso ao nascer, idade gestacional, idade da mãe, estado civil, profissão, número de filhos, escolaridade dos pais (mãe/pai), participação em programas sociais federais e estaduais como Bolsa Família, Benefício de Prestação Continuada e Leite das Crianças, e o diagnóstico clínico. Essa anamnese era específica para cada faixa etária (0-6 meses, 6-23 meses; 2-4 anos e maiores de cinco anos) em função das questões que abordavam o consumo alimentar.

A avaliação do consumo alimentar era realizada pelo método Recordatório de 24 horas (FISBERG; MARCHIONI; COLUCCI, 2009) embasado no material do Ministério da Saúde intitulado "Orientações para Avaliação de Marcadores de Consumo Alimentar na Atenção Básica” (BRASIL, 2015a), a partir do qual eram construídos indicadores de consumo, a saber: 
aleitamento materno exclusivo em menores de seis meses; para crianças de 6-23 meses: aleitamento materno continuado; introdução de alimentos; diversidade alimentar mínima; frequência mínima e consistência adequada; consumo de alimentos ricos em ferro; consumo de alimentos ricos em vitamina A; consumo de alimentos ultraprocessados; consumo de hambúrguer e/ou embutidos; consumo de bebidas adoçadas; consumo de macarrão instantâneo, salgadinhos de pacote ou biscoito salgados; consumo de biscoito recheado, doces ou guloseimas. Para crianças maiores de dois anos eram construídos os seguintes indicadores: hábito de realizar no mínimo as três refeições principais do dia; hábito de realizar as refeições assistindo à televisão; consumo de feijão; consumo de frutas; consumo de verduras e legumes; consumo de hambúrguer e/ou embutidos; consumo de bebidas adoçadas; consumo de macarrão instantâneo, salgadinhos de pacote ou biscoito salgados; consumo de biscoito recheado, doces ou guloseimas (BRASIL, 2015a).

Depois de traçado o diagnóstico nutricional antropométrico e alimentar de cada criança, as discentes sob supervisão direta dos docentes estabeleciam orientações chaves dependendo do EN e da condição social de cada criança e família. Na maioria das vezes, as orientações eram realizadas no momento dos atendimentos e as principais orientações eram repassadas às mães em uma folha escrita. Nos casos de doenças mais graves, como neuropatias (paralisia cerebral), crianças em uso de sondas enterais, ocorria a elaboração de planos alimentares e orientações mais específicas, pois essas crianças se apresentavam nutricionalmente mais comprometidas. Para aquelas crianças com necessidade de acompanhamento alimentar e nutricional, eram agendadas mensalmente consultas de retorno com a finalidade de verificar se houve melhora no EN antropométrico e alimentar das crianças e se as orientações alimentares estavam sendo seguidas. Caso essas não estivessem sendo seguidas eram readequadas ou reforçadas. Todas as orientações repassadas às mães seguiam os materiais do Ministério da Saúde e da Sociedade Brasileira de Pediatria (BRASIL, 2014; SOCIEDADE BRASILEIRA DE PEDIATRIA, 2012).

Além dos atendimentos ambulatoriais também foram realizadas atividades de educação alimentar e nutricional (EAN) como oficina culinária e roda de conversa com temáticas abrangentes ao público atendido, buscando melhorar o conhecimento acerca das práticas alimentares saudáveis para esta população.

O projeto de extensão foi aprovado pelo Comitê de Ética em Pesquisa da UNICENTRO (Ofício n. ${ }^{\circ}$ 079/2009), sendo realizado de acordo com as Normas e Diretrizes Éticas de Resolução do Conselho Nacional de Saúde do Ministério da Saúde, pois os dados coletados nos atendimentos também eram utilizados para fins de estudos e pesquisas. 


\section{RESULTADOS E ANÁLISES}

O Centro de Recuperação Nutricional atende crianças na faixa etária de 0 a 6 anos de idade com carências nutricionais, principalmente anemia ferropriva, desnutrição crônica representada pela baixa estatura, parasitoses intestinais e, algumas delas, doenças raras e neuropatias (SALDAN; BRECAILO, 2014). Apesar das carências nutricionais representarem a maioria dos atendimentos, também são atendidas crianças com sobrepeso e obesidade.

Desde o início desta etapa do projeto que iniciou em maio de 2015 até o mês de setembro de 2016 foram realizados 79 atendimentos iniciais e 54 atendimentos de retorno, totalizando 133 atendimentos. Os atendimentos de retorno aconteciam da mesma forma que os atendimentos iniciais, ou seja, de forma individual, no entanto a anamnese preenchida era reduzida, focando-se principalmente no EN antropométrico e alimentar da criança. Na consulta de retorno era observado se houve melhora ou não em relação ao atendimento inicial e o que poderia ser implementado para aquelas crianças que continuavam comprometidas nutricionalmente ou que as mães não conseguiam seguir as orientações sugeridas anteriormente.

A maioria das crianças nasceu a termo (37-41 semanas) e com peso adequado $(\geq 2.500$ gramas), as mães, em sua maioria, tinham entre 20 a 34 anos, residiam com companheiro, não trabalhavam fora e possuíam o ensino fundamental incompleto. Mais da metade das famílias estavam inscritas em programas sociais federal e/ou estadual de transferência de renda e de recebimento de alimentos. A relação dos atendimentos prestados segundo faixa etária das crianças pode ser vista na Figura 1.

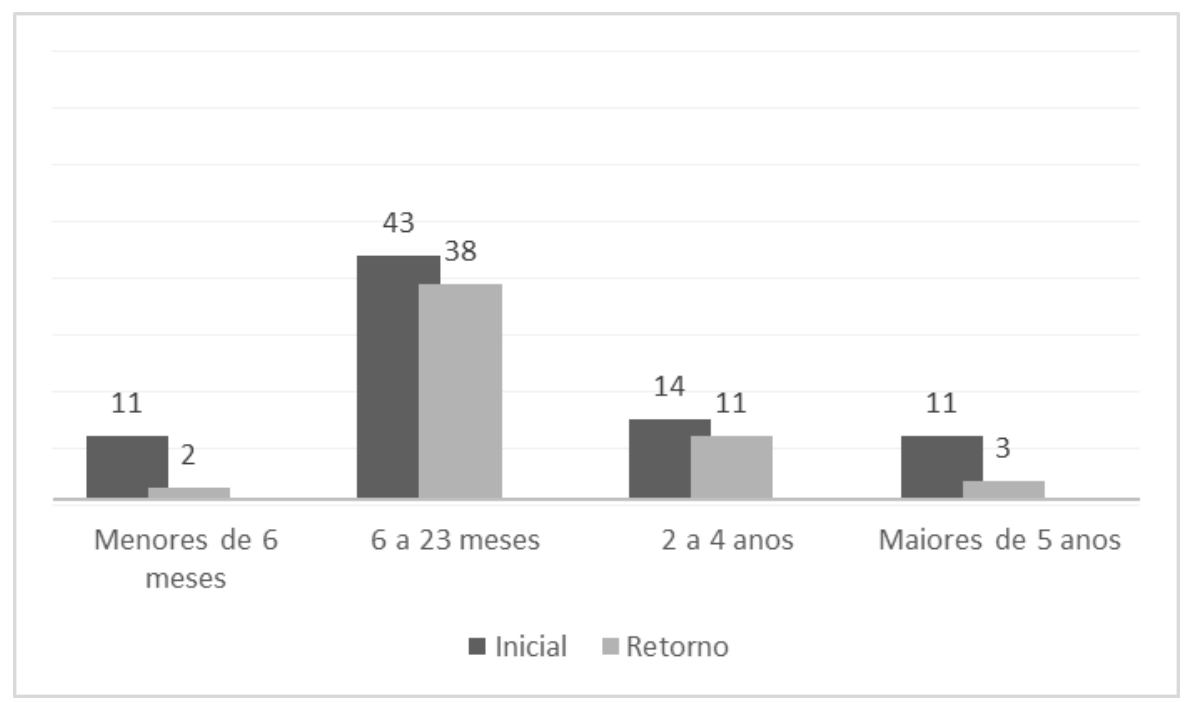

Figura 1. Número de atendimentos nutricionais ambulatoriais inicias e de retorno segundo faixa etária das crianças, Guarapuava-PR 2015-2016.

Fonte: elaborado pelas autoras. 
A faixa etária com maior número de atendimentos foi a de 6-23 meses, sendo que uma das hipóteses para que isso ocorra é que essa fase compreende o momento de introdução da alimentação complementar e corresponde à fase na qual as mães/responsáveis apresentam muitas dúvidas acerca do que a criança pode ou não receber. Também é uma fase onde aparecem carências nutricionais como anemia ferropriva (BRASIL, 2012). Para que esse processo transcorra de maneira tranquila, os cuidadores precisam ter um maior contato com profissionais capacitados a orientar o que as crianças necessitam em termos alimentares, sendo o nutricionista o profissional capacitado a realizar essa orientação (MARINHO et al., 2016). Entretanto, as faixas de menores de seis meses e de maiores de cinco anos apresentaram menor número de atendimentos.

Os indicadores de consumo alimentar das crianças de 6-23 meses apontaram que a introdução de alimentos e a diversidade alimentar mínima foi insuficiente e o consumo de alimentos ultraprocessados era maior do que 70\%, em especial as bebidas adoçadas. Um dado positivo refere-se ao consumo de alimentos ricos em ferro (superior a 80\%), em especial o feijão. Os indicadores de consumo alimentar para crianças maiores de 2 anos indicaram que mais de $70 \%$ faziam ao menos três refeições diárias, o feijão foi o alimento saudável mais consumido, porém as bebidas adoçadas, as bolachas recheadas, doces e guloseimas tiveram consumo elevado (mais de 60\%).

Dados da Pesquisa Nacional de Saúde realizada em 2013 mostram que já no início da vida as crianças recebem alimentos não recomendados, sendo que $60,8 \%$ das crianças menores de dois anos consumiam bolachas, biscoitos ou bolos e 32,3\% tomavam refrigerante ou suco artificial (BRASIL, 2015b).

O EN das crianças atendidas, segundo o índice Peso para Idade (P/I), está apresentado na Figura 2.

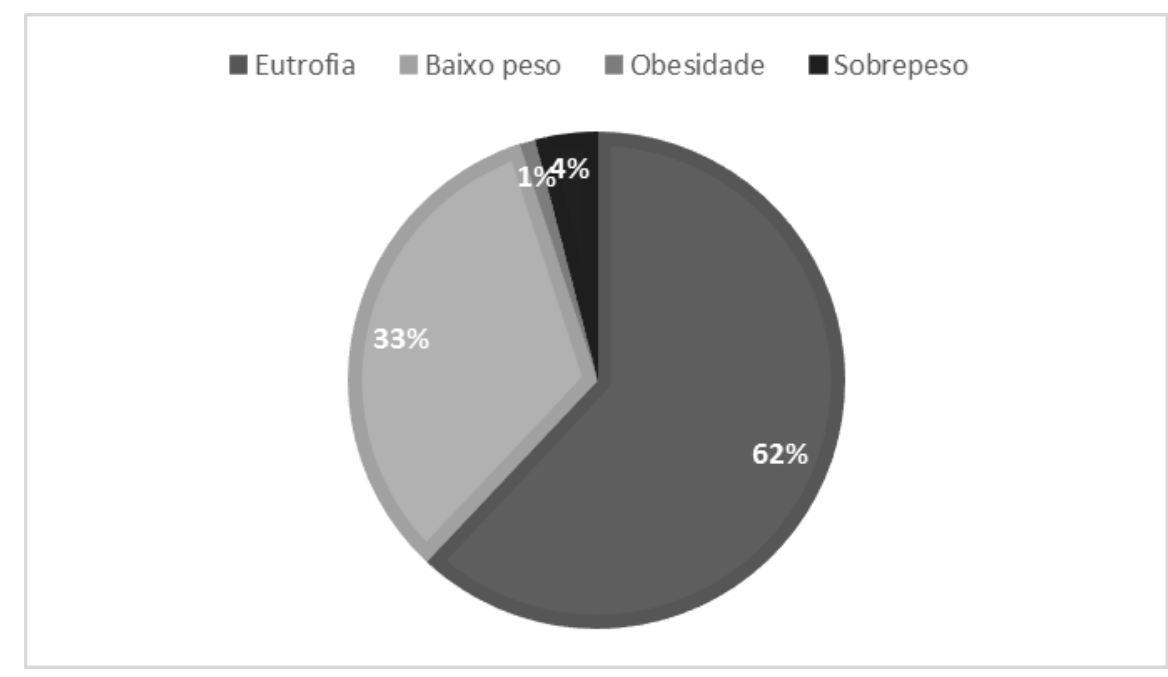


Figura 2. Estado nutricional segundo Peso/Idade das crianças atendidas em consulta inicial em Centro de Recuperação Nutricional de Guarapuava-PR, 2015-2016.

Fonte: elaborado pelas autoras.

Como pode ser observado, apesar da maioria das crianças apresentarem EN adequado no primeiro atendimento $(n=49,62 \%)$, observa-se que $38 \%(n=84)$ tinham o EN comprometido, sendo que 33\% $(\mathrm{n}=26)$ das crianças apresentaram baixo peso para a idade. Segundo Saldan et al. (2013) nos atendimentos prestados no Centro de Recuperação Nutricional nos anos de 2009 e 2010, 51,1\% das crianças tinham peso insuficiente para idade. No estudo de Silva et al. (2016) que avaliou o EN de 360 crianças, 51,7\% das crianças apresentaram eutrofia, 0,3\% baixo peso, 28\% risco de sobrepeso, 14,4\% sobrepeso e 5,6\% eram obesas. Já em um estudo realizado por Caldas et al. (2016) com crianças menores de um ano, idade em que foi realizado maior número de atendimentos no Centro de Recuperação Nutricional, pode-se observar que 91,5\% delas encontravam-se eutróficas quando avaliadas pelo índice Peso/Idade.

O comprometimento do EN de crianças, como evidenciado no presente estudo e demostrado também nos estudos de Souza, Pedraza e Menezes (2012) e Oliveira et al. (2007), associam-se a fatores de diferentes níveis de complexidade, a exemplo das condições socioeconômicas da família, do hábito alimentar dos pais e das características da própria criança. Por este motivo, os atendimentos são essenciais para se corrigir possíveis déficits nutricionais da população atendida, dar suporte às mães para que entendam o que pode ser melhorado na alimentação da criança e assim no seu EN, sempre respeitando os hábitos alimentares da família.

As atividades de EAN desenvolvidas no centro aconteceram após convite às mães participantes do projeto e constituíram uma oficina culinária sobre a introdução da alimentação complementar para mães e crianças com a elaboração de receitas de papa salgada e papa doce mostrando a evolução da consistência da alimentação infantil desde o início (a partir dos seis meses de vida) até chegar à alimentação da família (por volta de um ano de idade). Essa oficina culinária foi elaborada em virtude do número de crianças atendidas na faixa etária de 6-23 meses de idade ser relevante e dessa fase da vida ser primordial para a formação dos hábitos alimentares.

A outra atividade desenvolvida foi uma roda de conversa que teve como objetivo discutir a alimentação das crianças e das famílias, tendo como pano de fundo a temática do consumo de alimentos ultraprocessados e a quantidade de sal, açúcar e gorduras presentes nesses alimentos. A conversa foi conduzida pelas acadêmicas e docentes do projeto e o público participante dialogou sobre as práticas de alimentação das crianças e famílias no domicílio e as dificuldades encontradas para manter uma alimentação saudável. Essa atividade foi conduzida devido ao expressivo número de crianças que consumiam alimentos industrializados e ultraprocessados. As atividades tiveram duração média de duas horas com a participação de quinze mães. 
O presente projeto também se configura com um espaço para que os discentes possam conviver com a prática e a realidade profissional, bem como desenvolver pesquisas a partir dos atendimentos realizados, o que vai de encontro com uma das diretrizes da extensão que é a indissociabilidade do ensino, pesquisa e extensão.

Cabe ressaltar que apesar dos atendimentos nutricionais prestados à população de crianças e mães ser considerado positivo tanto pela direção e profissionais do Centro de Recuperação Nutricional quanto pelo público participante, tem-se um comparecimento do público de apenas $50 \%$ nos atendimentos agendados e esse número é ainda menor quando se trata de atendimentos de retorno.

\section{CONSIDERAÇÕES FINAIS}

Os atendimentos nutricionais ambulatoriais foram maiores na faixa etária dos 6-23 meses de idade e a maioria das crianças eram oriundas de famílias em situação social desfavorável. Apesar da maioria das crianças estar eutrófica, segundo o índice P/I, observou-se que 38\% tinham algum comprometimento do $\mathrm{EN}$, sendo fundamental seu acompanhamento. A introdução da alimentação complementar e a diversidade alimentar era insuficiente e o consumo de alimentos ultraprocessados elevado. As atividades de EAN foram desenvolvidas para auxiliar as mães nos aspectos da introdução da alimentação complementar saudável e visando a redução do consumo de alimentos ultraprocessados.

O desenvolvimento do presente projeto de extensão busca ao longo dos anos proporcionar adequados crescimento e desenvolvimento, bem como a construção de hábitos alimentares saudáveis pode prevenir carências nutricionais, inadequações do EN como desnutrição, sobrepeso, obesidade e doenças associadas. Além disso, o papel da universidade de levar o conhecimento científico para fora dos muros da instituição e atingir quem mais precisa desse conhecimento tem sido um ponto de destaque do projeto de extensão.

\section{AGRADECIMENTOS}

À Fundação Araucária de Apoio ao Desenvolvimento Científico e Tecnológico do Estado do Paraná pela concessão de duas bolsas de extensão para discentes de graduação dos Programas PIBIS e PIBEX. 
Atendimentos nutricionais ambulatoriais e estado nutricional de crianças menores de seis anos

\section{REFERÊNCIAS}

BLACK, R. E. et al. Maternal and child undernutrition: global and regional exposures and health consequences. Lancet, v. 371, p. 243-260, 2008.

BRASIL. Ministério da Saúde. Secretaria de Atenção à Saúde. Departamento de Atenção Básica. Orientações para avaliação de marcadores de consumo alimentar na Atenção Básica. Brasília: Ministério da Saúde, 2015a.

BRASIL. Ministério do Planejamento, Orçamento e Gestão. Instituto Brasileiro de Geografia e Estatística. Pesquisa nacional de saúde: 2013: ciclos de vida: Brasil e grandes regiões. Rio de Janeiro: IBGE, 2015b.

BRASIL. Ministério da Saúde. Secretaria de Atenção à Saúde. Departamento de Atenção Básica. Dez passos para uma alimentação saudável: guia alimentar para crianças menores de dois anos um guia para o profissional de saúde na atenção básica. 2. ed. 1. reimpr. Brasília, DF: Ministério da Saúde, 2014.

BRASIL. Ministério da Saúde. Secretaria de Atenção à Saúde. Departamento de Atenção Básica. Saúde da criança: crescimento e desenvolvimento. Brasília: Ministério da Saúde, 2012.

BRASIL. Ministério da Saúde. Secretaria de Atenção à Saúde. Departamento de Atenção Básica. Orientações para a coleta e análise de dados antropométricos em serviços de saúde: norma técnica do Sistema de Vigilância Alimentar e Nutricional - SISVAN. Brasília: Ministério da Saúde, 2011.

BROOKS, J. et al. Low weight, morbidity, and mortality in children with cerebral palsy: new clinical growth charts. Pediatrics, v. 128, n. 2, p. e299-e307, 2011.

CALDAS, D. R. C. et al. Aleitamento materno e estado nutricional de crianças menores de um ano de um município do Nordeste do Brasil. Ensaios e Ciência: Ciências Biológicas, Agrárias e da Saúde, Campo Grande, v. 20, n. 1, p. 3-10, 2016.

CARVALHO, M. F. et al. Acompanhamento do crescimento em crianças menores de um ano: situação nos serviços de saúde em Pernambuco, Brasil. Cadernos de Saúde Pública, Rio de Janeiro, v. 24, n. 3, p. 675-685, 2008.

COMITÊ CIENTÍFICO DO NÚCLEO CIÊNCIA PELA INFÂNCIA. Estudo I: o impacto do desenvolvimento na primeira infância sobre a aprendizagem. 2014. Disponível em: $<$ http://www.fmcsv.org.br/pt-br/acervo-digital/Paginas/o-impacto-no-desenvolvimento-daprimeira-infancia-sobre-a-aprendizagem.aspx $>$ Acesso em: 13 fev. 2017.

DUNCAN, B. B.; SCHMIDT, M. I.; GIUGLIANI, E. R. J. Medicina ambulatorial: condutas de atenção primária baseada em evidências. 3. ed. Porto Alegre: Artmed, 2004.

FENTON, T. R.; KIM, J. H. A systematic review and meta-analysis to revise the Fenton growth chart for preterm infants. BMC Pediatrics, v. 13, n. 59, 2013.

FISBERG, R. M.; MARCHIONI, D. M. L.; COLUCCI, A. C. A. Avaliação do consumo alimentar e da ingestão de nutrientes na prática clínica. Arquivos Brasileiros de Endocrinologia \& Metabologia, São Paulo, v. 53, n.5, p. 617-624, 2009. 
Atendimentos nutricionais ambulatoriais e estado nutricional de crianças menores de seis anos

MARINHO, L. M. F. et al. Situação da alimentação complementar de crianças entre 6 e 24 meses assistidas na Rede de Atenção Básica de Saúde Macaé, RJ, Brasil. Ciência \& Saúde Coletiva, Rio de Janeiro, v. 21, n. 3, p. 977-986, 2016.

MELLO, E. D.; LUFT, V. C.; MEYER, F. Obesidade infantil: como podemos ser eficazes? Jornal de Pediatria, Rio de Janeiro, v. 80, n. 3, p. 173-182, 2004.

MUSTACCHI, Z. Curvas padrão pôndero-estatural de portadores de Síndrome de Down procedentes da região urbana da cidade de São Paulo. 2002. 192 f. Tese (Doutorado em Farmácia) - Faculdade de Ciências Farmacêuticas da Universidade de São Paulo, São Paulo, 2002.

NASSER, L. A. Importância da nutrição, da infância à adolescência. In: FAGIOLI, D. Educação nutricional na infância e na adolescência: planejamento, intervenção, avaliação e dinâmicas. São Paulo: RCN Editora. p. 33-38, 2006.

OLIVEIRA, L. P. M. et al. Preditores do retardo de crescimento linear em pré-escolares: uma abordagem multinível. Cadernos de Saúde Pública, Rio de Janeiro, v. 23, n. 3, p. 601-613, 2007.

SALDAN, P. C.; BRECAILO, M. K. Atendimento ambulatorial de crianças em centro de recuperação nutricional. Revista Conexão UEPG, Ponta Grossa, v. 10, n. 1, p. 72-79, 2014.

SALDAN, P. C. et al. Perfil antropométrico, alimentação e situação socioeconômica de crianças atendidas em Centro de Recuperação Nutricional em Guarapuava, Paraná. Revista Baiana de Saúde Pública, Salvador, v. 37, n. 1, p. 205-221, 2013.

SIGULEM, D. M.; TADDEI, J. A. A. C. (Ed.). Nutrição e alimentação nos dois primeiros anos de vida. São Paulo: Compacta Nutrição, 2004.

SILVA, H. A. M. et al. Vigilância nutricional de crianças menores de dois anos do município de redenção, Ceará: a importância do diagnóstico para planejamento das políticas públicas nesse grupo etário. Revista Brasileira de Obesidade, Nutrição e Emagrecimento, São Paulo. v. 10, n. 56, p. 62-73, 2016.

SOCIEDADE BRASILEIRA DE PEDIATRIA. Departamento de Nutrologia. Manual de orientação para a alimentação do lactente, do pré-escolar, escolar, do adolescente e na escola. 3. ed. Rio de Janeiro: SBP, 2012.

SOUZA, M. M.; PEDRAZA, D. F.; MENEZES, T. N. Estado nutricional de crianças assistidas em creches e situação de insegurança alimentar de suas famílias. Ciência \& Saúde Coletiva, Rio de Janeiro, v. 17, n. 12, p. 3425-3436, 2012.

VIEIRA, R. W. et al. Do aleitamento materno à alimentação complementar: atuação do profissional nutricionista. Revista Acadêmica Saúde \& Ambiente, Duque de Caxias, v. 4, n. 2, p. 1-8, 2009.

Recebido em: 13/04/2017

Aceito em: 25/04/2018 